\title{
ON MONOTONE CONVERGENCE TO SOLUTIONS OF
}

$$
u^{\prime}=g(u, t)
$$

\section{RICHARD BELLMAN}

1. Introduction. The purpose of this brief note is to show that Newton's method of successive approximation to the solution of a functional equation yields a monotone increasing sequence of approximations to the solution of the first order differential equation,

$$
u^{\prime}=g(u, t), \quad u(0)=c,
$$

provided that $g(u, t)$ is uniformly convex in $u$ for $t$ in some fixed interval $\left[0, t_{0}\right]$.

The connection between the methods and results presented here and the theory of dynamic programming is treated in [1].

2. Newton's method of approximation. Write

$$
h(u, t)=\frac{\partial g}{\partial u}(u, t),
$$

and consider the system of equations

$$
\begin{aligned}
& \text { (a) } \frac{d u_{0}}{d t}=g\left(v_{0}, t\right)+\left(u_{0}-v_{0}\right) h\left(v_{0}, t\right), u_{0}(0)=c, \\
& \text { (b) } \frac{d u_{n+1}}{d t}=g\left(u_{n}, t\right)+\left(u_{n+1}-u_{n}\right) h\left(u_{n}, t\right), u_{n+1}(0)=c, \\
& n=0,1,2, \cdots,
\end{aligned}
$$

where $v_{0}(t)$ is a known function of $t$ which is taken to be continuous over $\left[0, t_{0}\right]$.

This is Newton's method of approximation applied to the differential equation of (1.1).

3. Montonicity of convergence. Let us now demonstrate the following result

THEOREM. Let $h(u, t)$ exist, be continuous, and monotone increasing in $u$ for $0 \leqq t \leqq t_{0}$. Then

$$
u_{0}(t) \leqq u_{1}(t) \leqq \cdots \leqq u_{n}(t) \leqq \cdots, 0 \leqq t \leqq t_{1},
$$

where $t_{1}>0$. The limit $u(t)=\lim _{n \rightarrow \infty} u_{n}(t)$ is the solution of (1.1).

Proof. We begin with the observation that the uniform convexity

Received by the editors March 13, 1957. 
of $g(u, t)$ yields the result that

$$
g(u, t)=\operatorname{Max}_{v}[g(v, t)+(u-v) h(v, t)]
$$

for $0 \leqq t \leqq t_{0}$.

Hence

$$
\begin{aligned}
\frac{d u_{n+1}}{d t} & =g\left(u_{n}, t\right)+\left(u_{n+1}-u_{n}\right) h\left(u_{n}, t\right) \\
& \leqq g(v, t)+\left(u_{n+1}-v\right) h(v, t),
\end{aligned}
$$

where $v=v(t)$ is the function which maximizes the function $g(u, t)$ $+\left(u_{n+1}-u\right) h(u, t)$. It follows that the solution of

$$
\frac{d w}{d t}=g(v, t)+(w-v) h(v, t), w(0)=c,
$$

majorizes the solution of

$$
\frac{d u_{n+1}}{d t}=g\left(u_{n}, t\right)+\left(u_{n+1}-u_{n}\right) h\left(u_{n}, t\right), u_{n+1}(0)=c,
$$

within a common interval of existence, i.e. $w(t) \geqq u_{n+1}(t)$.

Since the function $v(t)$ which maximizes is $u_{n+1}(t)$, we see that the solution of (4) is precisely the function $u_{n+2}(t)$.

This argument showed that $u_{1}(t) \geqq u_{0}(t)$ and, inductively, that (3.1) holds within a common interval of existence. That such an interval exists follows the usual lines, and similarly for the proof of convergence which is now equivalent to uniform boundedness of the sequence $\left\{u_{n}(t)\right\}$.

4. Multi-dimensional case. The proof presented above hinged on the fact that any function $u(t)$ satisfying the inequality

$$
\frac{d u}{d t} \leqq a(t) u+b(t), u(0)=c,
$$

is majorized by the solution of

$$
\frac{d v}{d t}=a(t) v+b(t), v(0)=c .
$$

The corresponding result for systems is not unreservedly true. If $\left\{x_{i}(t)\right\}$ is a set of functions satisfying

$$
\frac{d x_{i}}{d t} \leqq \sum_{j=1}^{n} a_{i j}(t) x_{j}+b_{i}(t), x_{i}(0)=c_{i}, \quad i=1,2, \cdots, n,
$$


it is not necessarily true that $x_{i}(t) \leqq y_{i}(t), i=1,2, \cdots, n, t \geqq 0$, where the $y_{i}(t)$ satisfy the equations

$$
\frac{d y_{i}}{d t}=\sum_{j=1}^{n} a_{i j}(t) y_{j}+b_{i}(t), y_{i}(0)=c_{i}, \quad i=1,2, \cdots, n .
$$

If, however, we have

$$
a_{i j}(t) \geqq 0, \quad t \geqq 0, \quad i \neq j,
$$

then the result does hold, cf. [2].

Consequently, if we take the Newton approximations

$$
\begin{aligned}
& \frac{d u_{n+1}}{d t}=f\left(u_{n}, v_{n}\right)+\left(u_{n+1}-u_{n}\right) \frac{\partial f}{\partial u_{n}}+\left(v_{n+1}-v_{n}\right) \frac{\partial f}{\partial v_{n}}, u_{n+1}(0)=c_{1}, \\
& \frac{d v_{n+1}}{d t}=g\left(u_{n}, v_{n}\right)+\left(u_{n+1}-u_{n}\right) \frac{\partial g}{\partial u_{n}}+\left(v_{n+1}-v_{n}\right) \frac{\partial g}{\partial v_{n}}, v_{n+1}(0)=c_{2},
\end{aligned}
$$

$n=0,1,2, \cdots$, with $u_{0}, v_{0}$ prescribed continuous functions, to the system

$$
\begin{array}{ll}
\frac{d u}{d t}=f(u, v), & u(0)=c_{1}, \\
\frac{d v}{d t}=g(u, v), & v(0)=c_{2},
\end{array}
$$

we can assert that

$$
u_{n} \leqq u_{n+1}, \quad v_{n} \leqq v_{n+1}, \quad t \geqq 0, n=0,1,2, \cdots,
$$

provided that

$$
\frac{\partial f}{\partial v} \geqq 0, \quad \frac{\partial g}{\partial u} \geqq 0,
$$

for all $u$ and $v$, and provided that $f(u, v)$ and $g(u, v)$ are strictly convex functions of $u$ and $v$.

\section{BIBLIOGRAPHY}

1. R. Bellman, Functional equations in the theory of dynamic programming, V, Positivity and quasi-linearity, Proc. Nat. Acad. Sci. U.S.A. vol. 41 (1955) pp. 743746.

2. R. Bellman, I. Glicksberg, and $\mathrm{O}$. Gross, On some variational problems occurring in the theory of dynamic programming, Rend. Circ. Mat. Palermo vol. III (1954) pp. 1-35.

RAND CORPORATION 